\title{
Heun-type solutions for Schwarzschild metric with electromagnetic fields
}

\author{
T. Birkandan ${ }^{1}$ and M. Hortaçsu ${ }^{2}$ \\ ${ }^{1}$ Istanbul Technical University - Department of Physics, Istanbul, Turkey \\ ${ }^{2}$ Mimar Sinan Fine Arts University - Department of Physics, Istanbul, Turkey
}

October 13, 2017

\begin{abstract}
We find confluent Heun solutions to the radial equations of two Halilsoy-Badawi metrics. For the first metric, we studied the radial part of the massless Dirac equation and for the second case, we studied the radial part of the massless Klein-Gordon equation.
\end{abstract}

\section{Introduction}

Heun functions [1, 2] seem to be still a novelty among theoretical physicists although they were introduced nearly 130 years ago. After the centennial conference which took place in 1989 and the papers presented in this conference were published in a book [2, there was an explosion of papers in this field [3]. Many equations whose exact solutions were not known turned out to have solutions in this set. After referring to people [4, 5, 6] who tried to show whether the exact solutions of the celebrated "Teukolsky Master Equation" [7 can be written down in terms of the confluent forms of the Heun equation, Batic and Schmidt 8] showed that the "Teukolsky master Equation" (and similar equations) could be transformed in any relevant type-D metric into a Heun form. Although the Heun equation and its confluent forms are much better known today in the theoretical physics community and included in some mathematical packages, we still find some authors who do not identify the equations they find properly.

Here we give two examples of metrics which yield confluent Heun solutions for the equations describing a test particle whose wave equation is written in the background metric of these metrics in the linear approximation, ignoring the backreaction and nonlinear terms in the Einstein-Maxwell equations. In the first example the massless Dirac equation and in the second, the massless Klein-Gordon equation are studied.

\section{Dirac equation}

In a very interesting paper 9] Al-Badawi and Owaidat study the Dirac equation in the background of the spherically symmetric solution of the Einstein- Maxwell equations, analogous to the Schwarzschild metric, in the presence of spherically symmetric static electromagnetic field.

For the metric they use a solution found by one of these authors with Halilsoy [10. Actually this metric was previously discovered by Ray and Wei[11. Metrics, when a Schwarzschild metric is in an homogeneous electromagnetic field were given by Halilsoy [12, 13], as stated in [14, and were included in the book by Griffiths and Podolsky [15]. This solution is a superposition of the Schwarzschild [16] solution with an external, stationary electromagnetic Bertotti-Robinson solution [17, 18]. 
The metric is given as

$$
\begin{gathered}
d s^{2}=\frac{r^{2}-2 M r}{r^{2} f(r)}\left[d t-M q\left(1+a^{2}\right) \cos \theta d \phi\right]^{2} \\
-\frac{r^{2} f(r)}{r^{2}-2 M r} d r^{2}-r^{2} f(r)\left(d \theta^{2}+\sin ^{2} \theta d \phi^{2}\right), \\
r^{2} f(r)=\frac{1}{2} r(r-2 M)\left[p\left(1+a^{2}\right)+a^{2}-1\right]+2 M a r+M^{2}\left[p\left(1+a^{2}\right)-2 a\right] .
\end{gathered}
$$

This is a type-D metric. $M$ is a constant parameter which mimics the role of the source mass in Newtonian approximations for the geodesics of test particles in the Schwarzschild metric when one considers large values of the luminosity radius $r ; p$ is the twisting parameter of the external electromagnetic field and $a$ is the interpolation parameter between two metrics used. This metric is in the class named as Plebanki and Demianski solutions[19].

When the parameter $a$ is zero one gets a metric which can be transformed into the Bertotti- Robinson solution by setting the twisting parameter to one. As stated in [15], in general this space time is a direct product of two two dimensional spaces of constant curvature, namely the 2-sphere and two- dimensional anti-de Sitter space-times. When $a$ is between zero and one, it contains a family of expanding Schwarzschild-Reissner-Nordstrom-de Sitter metrics. Here, as in [11, 10] we take the cosmological constant equal to zero.

The authors consider only a spinor test particle, in the linear approximation, ignoring the backreaction and nonlinear terms in the Einstein-Maxwell equations. They use the Newman-Penrose formalism 20 to separate the Dirac equation into radial and angular $(\theta)$ parts after assuming a periodic solution for the variables $t$ and $\phi$ which are along Killing directions. They can solve the angular equation in terms of the associated Legendre functions. For the radial equation, they use the WKB approximation and write their solutions in terms of exponentials.

We just considered the case in which the coupled spinor test particle has zero mass. When the test particle is massive, we could not obtain the exact solution in terms of known functions. For the massless case, the radial equations are of the form [9]:

$$
\begin{aligned}
& \frac{d T_{1}}{d r}+i \frac{k R^{2}}{H^{2} R^{2}} T_{1}=\frac{\lambda}{R H} T_{2}, \\
& \frac{d T_{2}}{d r}-i \frac{k R^{2}}{H^{2} R^{2}} T_{2}=\frac{\lambda}{R H} T_{1} .
\end{aligned}
$$

Here $\lambda$ is the eigenvalue, $R^{2}=r^{2} f(r)$ and

$$
H^{2}=\frac{r^{2}-2 M r}{r^{2} f(r)} .
$$

The exact solutions for the radial equations above turn out to be confluent Heun functions multiplied by exponentials and powers of the finite regular singular points of the radial equation. This is no surprise, since as Philipp and Perlick pointed out [21, Leaver 22, 5] and Fiziev [23, 24, 25] showed that the solution of a particle in the Schwarzschild background results in confluent Heun type solutions. In this respect, it looks like the solutions of the Eguchi-Hanson instanton [26] trivially extended to five dimensions [27. In this case, too, the angular equation has a solution which is in the family of hypergeometric functions, whereas the radial equation's solution is again a confluent Heun function [1, 2.

In the paper of Al-Badawi and Owaidat [9, we could not find the explicit expressions for the frequency $\omega$, the essential parameter in the WKB approximation, aside from an integral, to check the behavior of the incoming and outgoing waves as well as the expressions for the transition and reflection coefficients are not given. The authors end their paper by studying the plots of the effective potentials for different values of the parameters in the effective potential.

One can show that the radial equation can indeed be solved in terms of confluent Heun functions. This solution has regular singularities at $r=0$ and $r=2 M$ where the horizon is, and an irregular singularity at infinity. We first study the solution for $0<r<2 M$, assuming that the given metric is also valid here. We are aware of the fact that the metric used is only valid outside the event horizon due to the properties of the Schwarzschild metric. We do 
this calculation just to compare our solution with that given in 9 and see if it has the correct behavior around the regular singular points of the differential equation.

We did not give the second order equation obtained from the system of first order equations given above for the sake of brevity of the paper, since that equation is quite long. We, however, give a similar equation when we study the region for $r$ greater than $2 M$, the domain of $r$ in which we are really interested in this paper.

We only take the first solution which is analytic around the singularity at $r=0$. It reads as:

$$
\begin{aligned}
T_{1}(r)= & \mathrm{e}^{i / 2\left((p+1) a^{2}+p-1\right) r k} r^{i / 2 k\left(a^{2} p-2 a+p\right) M}(2 M-r)^{1 / 2+i / 2 k\left(a^{2} p+2 a+p\right) M} \times \\
& H_{C}\left(2 i M\left((p+1) a^{2}+p-1\right) k,-1 / 2+i k\left(a^{2} p-2 a+p\right) M,\right. \\
& 1 / 2+i k\left(a^{2} p+2 a+p\right) M,(4 M a k+i) M\left((p+1) a^{2}+p-1\right) k, \\
& 1 / 2 k^{2} M^{2}\left(a^{2}+1\right)^{2} p^{2} \\
& -1 / 2 M\left(a^{2}+1\right)\left(-2 M a^{2} k+4 M a k+2 k M+i\right) k p-2 k^{2} a^{3} M^{2} \\
& \left.-1 / 2 M k(-4 k M+i) a^{2}+M(2 k M+i) k a+i / 2 k M-\lambda^{2}+3 / 8, \frac{r}{2 M}\right),
\end{aligned}
$$

where $H_{C}$ denotes the confluent Heun function. We note that although this solution is analytic around $r=0$, it is not analytic around $r=2 M$. This is expected, since our solution is only a local solution which is analytic only in the neighborhood of one singularity [28]. Here the parameters are defined in [9].

These equations also have a second solution:

$$
\begin{aligned}
T_{1_{2}}(r)= & \mathrm{e}^{i / 2 r k\left((p+1) a^{2}+p-1\right)} r^{1 / 2-i / 2 k\left(a^{2} p-2 a+p\right) M}(2 M-r)^{1 / 2+i / 2\left(a^{2} p+2 a+p\right) k M} \times \\
& H_{C}\left(2 i M\left((p+1) a^{2}+p-1\right) k, 1 / 2-i k\left(a^{2} p-2 a+p\right) M,\right. \\
& 1 / 2+i\left(a^{2} p+2 a+p\right) k M, M\left((p+1) a^{2}+p-1\right)(4 k M a+i) k, \\
& 1 / 2 M^{2} k^{2}\left(a^{2}+1\right)^{2} p^{2} \\
& -1 / 2 M\left(a^{2}+1\right)\left(-2 M a^{2} k+4 k M a+2 k M+i\right) k p-2 k^{2} a^{3} M^{2} \\
& \left.-1 / 2 M(-4 k M+i) k a^{2}+M(2 k M+i) k a+i / 2 M k-\lambda^{2}+3 / 8, \frac{r}{2 M}\right) .
\end{aligned}
$$

In the following, we do not consider it, since it has a square root irregularity at $r=0$, our point of expansion.

The standard form of the confluent Heun equation is given as [29, 30]

$$
\frac{d^{2} H_{C}}{d z^{2}}+\left(\alpha+\frac{\gamma+1}{z-1}+\frac{\beta+1}{z}\right) \frac{d H_{C}}{d z}+\left(\frac{\mu}{z}+\frac{\nu}{z-1}\right) H_{C}=0,
$$

with solution $H_{C}(\alpha, \beta, \gamma, \delta, \eta, z)$, and the parameters have the relations

$$
\begin{gathered}
\delta=\mu+\nu-\alpha\left(\frac{\beta+\gamma+2}{2}\right), \\
\eta=\frac{\alpha(\beta+1)}{2}-\mu-\frac{\beta+\gamma+\beta \gamma}{2} .
\end{gathered}
$$

Since we are interested in the region $r>2 M$, outside the event horizon, the solution we gave above does not suit our purposes if we want to investigate the behavior of the wave for $r>2 M$. To find a solution to suit our purpose, we have to transform to the variable $u=r-2 M$. This will give us one solution which is analytic around $r=2 M$. This solution may not be analytic around $r=0$. Since we are not interested in the region $0<r<2 M$, this will not cause any problems.

From the two first order differential equations given above, equations [3] and [4], we derive a second order equation for $F_{1}$. This equation reads

$$
\left.A \frac{\mathrm{d}^{2}}{\mathrm{~d} u^{2}} T_{1}(u)+B \frac{\mathrm{d}}{\mathrm{d} u} T_{1}(u)+(C+D+E)\right) T_{1}(u)=0,
$$


where

$$
\begin{gathered}
A=(u+2 M)^{2} u^{2}, \\
B=(M+u)(u+2 M) u, \\
C=\left[\left((p / 2+1 / 2) a^{2}+p / 2-1 / 2\right)(u+2 M)^{2}-\left((p+1) a^{2}-2 a+p-1\right) M(u+2 M)\right. \\
\left.+M^{2}\left(a^{2} p-2 a+p\right)\right]^{2} k^{2}, \\
D=\left[\left((i / 2+i / 2 p) a^{2}+i / 2 p-i / 2\right)(u+2 M)^{3}-3 / 2 i M\left((p+1) a^{2}+p-1\right)(u+2 M)^{2}\right. \\
\left.+i(a-1) M^{2}(a+1)(u+2 M)+i M^{3}\left(a^{2} p-2 a+p\right)\right] k, \\
E=\lambda^{2}(u+2 M) u .
\end{gathered}
$$

Here we again take the solution which is analytic around $u=0(r=2 M)$, namely

$$
\begin{aligned}
T_{1}(u)= & \mathrm{e}^{-i / 2 u k\left((p+1) a^{2}+p-1\right)}(u+2 M)^{i / 2 k\left(a^{2} p-2 a+p\right) M} u^{-i / 2\left(a^{2} p+2 a+p\right) k M} \times \\
& H_{C}\left(2 i M\left((p+1) a^{2}+p-1\right) k,-1 / 2-i\left(a^{2} p+2 a+p\right) k M,\right. \\
& -1 / 2+i k\left(a^{2} p-2 a+p\right) M,-M\left((p+1) a^{2}+p-1\right)(4 k M a+i) k, \\
& 1 / 2 M^{2} k^{2}\left(a^{2}+1\right)^{2} p^{2}+1 / 2 M\left(a^{2}+1\right)\left(2 M a^{2} k+4 k M a-2 M k+i\right) k p \\
& \left.+2 k^{2} a^{3} M^{2}+1 / 2 M(4 M k+i) k a^{2}+M k(-2 M k+i) a-i / 2 M k-\lambda^{2}+3 / 8,-\frac{u}{2 M}\right) .
\end{aligned}
$$

The second solution is given below:

$$
\begin{aligned}
T_{1_{2}}(u)= & \mathrm{e}^{-i / 2 u k\left((p+1) a^{2}+p-1\right)}(u+2 M)^{i / 2 k\left(a^{2} p-2 a+p\right) M} u^{1 / 2+i / 2\left(a^{2} p+2 a+p\right) k M} \times \\
& H_{C}\left(2 i M\left((p+1) a^{2}+p-1\right) k, 1 / 2+i\left(a^{2} p+2 a+p\right) k M,\right. \\
& -1 / 2+i k\left(a^{2} p-2 a+p\right) M,-M\left((p+1) a^{2}+p-1\right)(4 k M a+i) k, \\
& 1 / 2 M^{2} k^{2}\left(a^{2}+1\right)^{2} p^{2}+1 / 2 M\left(a^{2}+1\right)\left(2 M a^{2} k+4 k M a-2 k M+i\right) k p \\
& \left.+2 k^{2} a^{3} M^{2}+1 / 2 M(4 k M+i) k a^{2}+k M(-2 k M+i) a-i / 2 M k-\lambda^{2}+3 / 8,-\frac{u}{2 M}\right) .
\end{aligned}
$$

We discard it since it has a second root non analyticity at $u=0$, our point of expansion.

Using $p=10, k=0.2, a=0.1, \lambda=0.7$ and $M=5$, we give the plots of the first solution for $0<r<2 M$ and $u>0$ in Figure 1 and Figure 2, respectively.

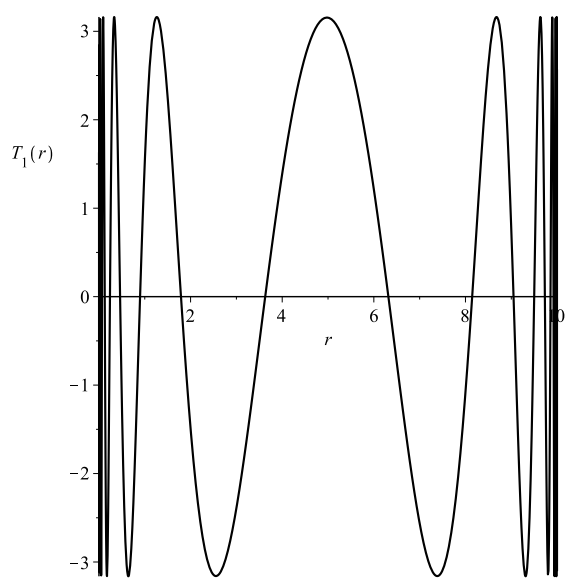

Figure 1: Solution between $0<r<2 M$ 


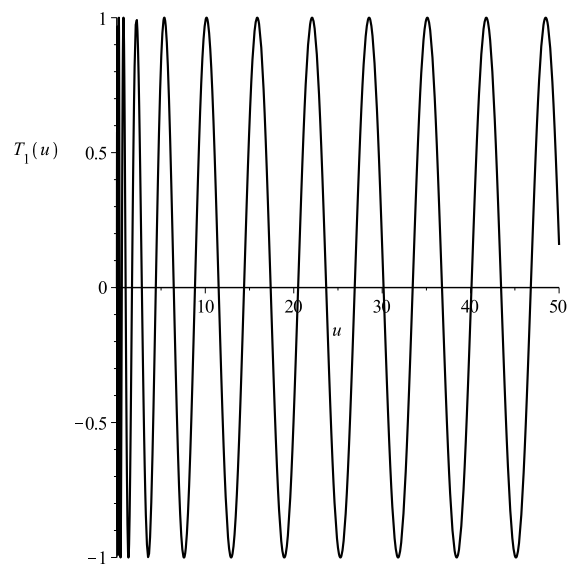

Figure 2: Solution for $u>0$

Polynomial solutions can be given for the confluent Heun equation under some conditions [30, 31. The identity $\mu+\nu=-N \alpha, N$ being the degree of the polynomial solution, should be satisfied along with a vanishing determinant. However, this identity is not useful in this case as $\mu+\nu=0$.

\section{Solution around infinity}

Solution of the confluent Heun equation around the irregular singularity at infinity can be given by the Thomé solution as 32 .

$$
\lim _{z \rightarrow \infty} U(z) \sim e^{ \pm i \omega z} z^{\mp i \eta-\left(B_{2} / 2\right)} .
$$

Note that this is just the first term in a nonconverging series, which must be considered as just an asymptotic one.

The confluent Heun equation is written in the form

$$
z(z-1) \frac{d^{2} U}{d z^{2}}+\left(B_{1}+B_{2} z\right) \frac{d U}{d z}+\left[B_{3}-2 \eta \omega(z-1)+\omega^{2} z(z-1)\right] U=0,
$$

for $\omega \neq 0$ and all other parameters are constants. This form is called the generalized spheroidal wave equation and finding the correspondence with the general form given by the equation (8) needs some algebra as the solutions are not in the same form (i.e. we need to define $V(z)=e^{i \omega z} U(z)$ first and then proceed with the solution). Studying the solution for our case, we find the first term in this asymptotic series as

$$
\lim _{u \rightarrow \infty} T_{1}(u) \sim e^{\left(2 i\left[(p+1) a^{2}+p-1\right]\right) M k u},
$$

as the solution around infinity. There is a second solution which behaves as $u^{4 i k M a-1}$, i.e. it vanishes as $u$ goes to infinity.

As to the physical interpretation of our solutions, we can get information only by plotting our solutions, since the general behavior of Heun functions is not generally known explicitly. We see the approach to singularity at $r=2 M$ when we plot our function for the range $0<r<2 M$. When we expand around one of the regular singular points, we expect such a behaviour around the second singular point.

To our surprise, the regular solution resembles a plane wave for $u>0$ (Figure 2) with almost constant frequency and constant amplitude. This was a surprise, since this is the behaviour only in the asymptotic region for the quasi-classical solution in reference [9]. The irregular solution, besides being non analytic at $u=0$, goes to zero, oscillating with vanishing amplitude. This behaviour is reflected in the behaviour of the second solution (asymptotic solution) which behaves as a reciprocal power of $u$. 


\section{Klein-Gordon equation}

In another recent paper by Al-Badawi, the Dirac equation is studied in a Schwarzschild black hole immersed in an electromagnetic universe with charge coupling 14 . Here the electromagnetic radiation is not attributed to the parameter M. This solution again interpolates the Schwarzschild [16] and Bertotti-Robinson [17, 18] solutions [14]. The metric is

$$
d s^{2}=\frac{\Delta}{r^{2}} d t^{2}-\frac{r^{2}}{\Delta} d r^{2}-r^{2}\left(d \theta^{2}+\sin ^{2} \theta d \phi^{2}\right),
$$

where $\Delta=r^{2}-2 M r+M^{2}\left(1-a^{2}\right)$ [33, 34]. Here, $M$ is the parameter used in the Schwarzschild solution and $a$ $(0<a \leq 1)$ is the external parameter. This metric has two horizons, the outer horizon at $r_{1}=M(1+a)$ and the inner horizon at $r_{2}=M(1-a)$. The external electromagnetic field shrinks at the outer horizon and expands at the inner horizon [33. Note that the $a=0$ case can be transformed into the Bertotti-Robinson solution [34].

We study the massless Klein-Gordon equation in the background of this metric, since we can not identify the solution for the massive case.

$$
\frac{1}{\sqrt{-g}} \partial_{\mu}\left(\sqrt{-g} g^{\mu \nu} \partial_{\nu} \Phi\right)=0
$$

in this background, namely,

$$
\Phi \sin ^{2} \theta r^{4} \omega^{2}+\Delta^{2} \sin ^{2} \theta \frac{\partial^{2} \Phi}{\partial r^{2}}+\Delta\left(\frac{\partial \Phi}{\partial r}\right) \sin ^{2} \theta \frac{\mathrm{d} \Delta}{\mathrm{d} r}-\Delta n^{2} \Phi+\Delta\left(\frac{\partial \Phi}{\partial \theta}\right) \sin \theta \cos \theta+\Delta \sin ^{2} \theta \frac{\partial^{2} \Phi}{\partial \theta^{2}}=0 .
$$

This equation can be separated into the radial and angular parts with the Ansatz

$$
\Phi=e^{-i \omega t} e^{i n \phi} F(r) S(\theta) .
$$

After defining the separation constant $\lambda$, the radial and angular parts are obtained as

$$
\begin{aligned}
& \frac{\mathrm{d}^{2} F(r)}{\mathrm{d} r^{2}}-\frac{F(r) \lambda}{\Delta}+\frac{F(r) r^{4} \omega^{2}+\Delta\left(\frac{\mathrm{dF}(\mathrm{r})}{\mathrm{d} r}\right) \frac{\mathrm{d} \Delta}{\mathrm{d} r}}{\Delta^{2}}=0, \\
& \frac{\mathrm{d}^{2} S(\theta)}{\mathrm{d} \theta^{2}}+S(\theta) \lambda+\frac{\left(\frac{\mathrm{d} S(\theta)}{\mathrm{d} \theta}\right) \sin \theta \cos \theta-S(\theta) n^{2}}{\sin ^{2} \theta}=0 .
\end{aligned}
$$

The angular part is in the form of the associated Legendre equation and the radial part can be solved in terms of confluent Heun functions. We change our parameter $r$ to $u=r-r_{1}, r_{1}$ being the outer event horizon in order to study the behavior outside the event horizons. We note that the event horizon is located at $r_{1}=M(1+a)$ and the inner horizon is located at $r_{2}=M(1-a)$. The radial solution is

$$
\begin{aligned}
F(u)= & \mathrm{e}^{-i \omega u} u^{\frac{i r_{1}{ }^{2} \omega}{r_{1}-r_{2}}}\left(u+r_{1}-r_{2}\right)^{\frac{i r_{2}{ }^{2} \omega}{r_{1}-r_{2}}} \times \\
& H_{C}\left(2 i \omega\left(r_{1}-r_{2}\right), \frac{2 i r_{1}{ }^{2} \omega}{r_{1}-r_{2}}, \frac{2 i r_{2}{ }^{2} \omega}{r_{1}-r_{2}},\left(-2 r_{1}{ }^{2}+2 r_{2}{ }^{2}\right) \omega^{2},\right. \\
& \left.\frac{2 r_{1}{ }^{4} \omega^{2}-4 r_{1}{ }^{3} \omega^{2} r_{2}-\lambda r_{1}{ }^{2}+2 r_{1} r_{2} \lambda-\lambda r_{2}{ }^{2}}{\left(r_{1}-r_{2}\right)^{2}},-\frac{u}{r_{1}-r_{2}}\right),
\end{aligned}
$$

and the second solution, namely

$$
\begin{aligned}
F_{2}(u)= & \mathrm{e}^{-i \omega u} u^{\frac{-i r_{1}{ }^{2} \omega}{r_{1}-r_{2}}}\left(u+r_{1}-r_{2}\right)^{\frac{i r_{2}{ }^{2} \omega}{r_{1}-r_{2}}} \times \\
& H_{C}\left(2 i \omega\left(r_{1}-r_{2}\right), \frac{-2 i r_{1}{ }^{2} \omega}{r_{1}-r_{2}}, \frac{2 i r_{2}{ }^{2} \omega}{r_{1}-r_{2}},\left(-2 r_{1}^{2}+2 r_{2}^{2}\right) \omega^{2},\right. \\
& \left.\frac{2 r_{1}^{4} \omega^{2}-4 r_{1}^{3} r_{2} \omega^{2}-r_{1}^{2} \lambda+2 r_{1} r_{2} \lambda-r_{2}{ }^{2} \lambda}{\left(r_{1}-r_{2}\right)^{2}},-\frac{u}{r_{1}-r_{2}}\right)
\end{aligned}
$$

These solutions may be interpreted as two waves with different phases, but both moving in the same direction asymptotically, since for large values of $u, \ln (u)$ is much smaller than $u$.

We just wanted to state that this test particle in this metric, too, has a Heun family solution. We will study other properties of this solution in further papers. 


\section{Conclusion}

Here we studied two different metrics given by [10] and [33. In the first case we studied the Dirac equation given in 9 and found that the radial solution can be expressed in terms of confluent Heun functions. We found the same structure in the second metric case [14, 33] for the Klein-Gordon equation.

\section{$5 \quad$ Acknowledgement}

We thank the anonymous referee for correcting our "careless" use of the physical and mathematical terminology. M.H. thanks Prof. Ibrahim Semiz for providing important literature and Prof. Nadir Ghazanfari for technical assistance. He also thanks the Science Academy, Turkey for support. This work is supported by TUBITAK, the Scientific and Technological Council of Turkey.

\section{References}

[1] Heun K., Math. Annalen 33 (1889) 161.

[2] Ronveaux A. (ed.), Heun's Differential Equations Oxford University Press (1995)

[3] Hortacsu M, Heun Functions and their uses in Physics in Proceedings of the 13th Regional Conference on Mathematical Physics, Antalya, Turkey, October 27-31, 2010, U. Camc1 and I. Semiz (eds.), pp. 23-39, World Scientific, Singapore (2013) (arXiv:1101.0471).

[4] Blaudin J., Poons R. and Marcilhacy G., Lett. Nuovo Cim. 38 (1983) 561.

[5] Leaver E.W., J. Math. Phys. 27 (1986) 1238.

[6] Suzuki H., Takasugi E. and Umetsu H., Prog.Theor.Physics 100 (1998) 491.

[7] Teukolsky S.A., Physical Review Letters 29 (1972) 1114.

[8] Batic D., Schmidt H., J.Math.Phys. 48 (2007) 042502.

[9] Al-Badawi A. and Owaidat M. Q., Gen. Relativ. Gravit. 49 (2017) 110 (arXiv:1702.00368).

[10] Halilsoy M., Al-Badawi A., Class. Quantum Grav. 12 (1995) 3013.

[11] Ray J.M., and Wei M.S., Il Nuovo Cimento, 42B (1977) 151.

[12] Halilsoy, M, Gen. Relativ. Gravit. 25 (1993) 275.

[13] Halilsoy, M, Gen. Relativ. Gravit. 25 (1993) 975.

[14] Al-Badawi A., arXiv:1702.01380 (2017).

[15] Griffiths J.B. and Podolsky J., Exact Space-Times in Einstein's General Relativity, Cambridge University Press (2009), p. 320.

[16] Schwarzschild K., Sitzungsber. K. Preuss. Akade. Wiss. 7 (1916) 189.

[17] Bertotti B., Phys. Rev. 116 (1959) 1331.

[18] Robinson I., Bull. Acad. Pol. Sci. 7 (1959) 351.

[19] Plebanski, J. and Demianski, M., Ann.Phys. (N.Y.) 98 (1986) 98.

[20] Newman E. T., Penrose R., J. of Math. Phys. 3 (1962) 566.

[21] Philipp D., Perlick V., arXiv:1503.08101 [gr-qc] (2015). 
[22] Leaver E., Proc. R. Soc. Lond. A 402 (1985) 285.

[23] Fiziev P., arXiv:gr-qc/0603003 (2006).

[24] Fiziev P., Class. Quant. Grav. 23 (2006) 2447.

[25] Fiziev P., J. Phys. Conference Series 66 (2007) 012016.

[26] Eguchi T., Hanson A.J., Physics Letters B 74 (1978) 249.

[27] Birkandan T., Hortacsu M., J.Math.Phys. 49 (2008) 054101.

[28] Arscott F.M, Ronveaux A. (ed.), Heun's Differential Equations Oxford University Press (1995), p 31.

[29] Fiziev P., Class. Quant. Grav. 27 (2010) 135001.

[30] Fiziev P.: J. Phys. A: Math. Theor. 43 (2010) 035203.

[31] Ciftci H., Hall R. L., Saad N., Dogu E., J. Phys. A: Math. Theor. 43 (2010) 415206.

[32] El-Jaick L.J., Figueiredo B.D.B., J. Math. Phys. 49 (2008) 083508.

[33] Halilsoy M., Al-Badawi A., Il Nuovo Cimento B 113 (1998) 761.

[34] Ovgun A., Int. J. Theor. Phys. 55 (2016) 2919 (arXiv:1508.04100). 